\title{
Papers
}

\section{Prophylactic treatment of migraine with angiotensin converting enzyme inhibitor (lisinopril): randomised, placebo controlled, crossover study}

\author{
Harald Schrader, Lars Jacob Stovner, Grethe Helde, Trond Sand, Gunnar Bovim
}

\begin{abstract}
Objective To determine the efficacy of an angiotensin converting enzyme inhibitor in the prophylaxis of migraine.

Design Double blind, placebo controlled, crossover study.

Setting Neurological outpatient clinic.

Participants Sixty patients aged 19-59 years with migraine with two to six episodes a month.

Interventions Treatment period of 12 weeks with one $10 \mathrm{mg}$ lisinopril tablet once daily for one week then two $10 \mathrm{mg}$ lisinopril tablets once daily for 11 weeks, followed by a two week wash out period. Second treatment period of one placebo tablet once daily for one week and then two placebo tablets for 11 weeks. Thirty participants followed this schedule, and 30 received placebo followed by lisinopril.

Main outcome measures Primary end points: number of hours with headache, number of days with headache, number of days with migraine. Secondary end points: headache severity index, use of drugs for symptomatic relief, quality of life and number of days taken as sick leave, acceptability of treatment.

Results In the 47 participants with complete data, hours with headache, days with headache, days with migraine, and headache severity index were significantly reduced by $20 \%(95 \%$ confidence interval $5 \%$ to $36 \%$ ), $17 \%$ (5\% to $30 \%$ ), $21 \%$ (9\% to $34 \%$ ), and $20 \%$ (3\% to $37 \%)$, respectively, with lisinopril compared with placebo. Days with migraine were reduced by at least $50 \%$ in 14 participants for active treatment versus placebo and 17 patients for active treatment versus run-in period. Days with migraine were fewer by at least $50 \%$ in 14 participants for active treatment versus placebo. Intention to treat analysis of data from 55 patients supported the differences in favour of lisinopril for the primary end points. Conclusion The angiotensin converting enzyme inhibitor, lisinopril, has a clinically important prophylactic effect in migraine.
\end{abstract}

\section{Introduction}

Despite treatment of symptomatic migraine with triptans many patients experience only partial relief of symptoms. Furthermore, about $30-40 \%$ do not respond, and in some, triptans induce headache. For these patients and for those who do not respond to non-specific treatments, prophylactic drugs are indicated for people who experience two or more attacks a month. Some $\beta$ blockers and the anti-epileptic drug sodium valproate have shown some prophylactic effect. There is some evidence for the efficacy of the 5-hydroxytryptamine receptor antagonists pizotifen and methysergide as well as for flunarizine and several non-steroidal anti-inflammatory drugs. Most of the recommended drugs, however, cause adverse events that preclude long term treatment. Thus, there is a need for new prophylactic drugs that have greater efficacy and are better tolerated.

We had observed an impressive improvement in migraine in a patient treated with lisinopril for hypertension and subsequently discovered a pilot study of angiotensin converting enzyme inhibitors and migraine. ${ }^{1}$ We also obtained anecdotal evidence for the efficacy of lisinopril in 10 women who had migraine, eight of whom reported fewer attacks during treatment. We therefore carried out a randomised, double blind, placebo controlled, crossover study to investigate the prophylactic effect of the angiotensin converting enzyme inhibitor lisinopril.

\section{Participants and methods}

The study followed the guidelines recommended by the International Headache Society's committee on clinical trials in migraine ${ }^{2}$ and was carried out between April 1998 and December 1999. Of the 60 randomised patients, 35 were recruited from an outpatient clinic and 25 responded to advertisements in a local newspaper.

Inclusion criteria were diagnosis of migraine with and without aura according to the criteria of the International Headache Society, ${ }^{3}$ men and women aged between 18 and 60 years, presence of migraine for more than a year, onset of migraine before the age of 50 years, and attacks of migraine occurring two to six times a month. Exclusion criteria were interval headache that the patient was unable to differentiate from migraine, use of prophylactic drugs for migraine in the four weeks before randomisation, pregnancy or inability to use contraceptives, decreased renal or hepatic function, hypersensitivity to angiotensin

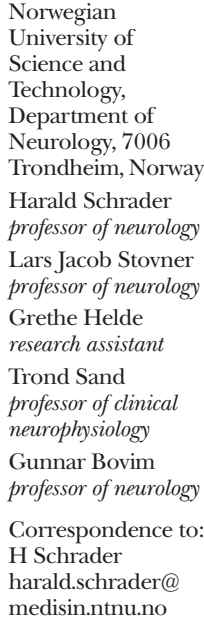

Norwegian University of Science and Technology, Department of Neurology, 7006 Trondheim, Norway Harald Schrader professor of neurology Lars Jacob Stovner professor of neurology Grethe Helde research assistant Trond Sand professor of clinical neurophysiology Gunnar Bovim professor of neurology

Correspondence to: H Schrader

harald.schrader@ medisin.ntnu.no

BMJ 2001;322:1-5 
converting enzyme inhibitors, history of angioneurotic oedema, and psychiatric disorder. The study was performed in accordance with the Declaration of Helsinki. The study protocol was approved by the regional ethics committee for medical research, and all patients gave written, informed consent before enrolment.

\section{Study design}

Participants who satisfied the inclusion criteria as judged by their case histories entered a four week placebo run-in period to verify the frequency of attacks. Participants were instructed to take one tablet daily and told that they would continue in the study only if the headache diary in this period showed two to six migraine attacks. All tablets for this study were supplied as round, white tablets containing either 10 mg lisinopril (active) or placebo (inactive). Active and inactive tablets were identical in appearance and were packed in identical bottles that were labelled with the patient number and appropriate period of the study. This ensured that both the patient and the investigator were unaware of the treatment that the participant was taking during the double blind treatment periods; during the run-in and wash out periods the investigator was aware that placebo treatment was being taken. The investigator was supplied with a sealed code for each individual patient that would be opened in case of an emergency that required knowledge of the treatment being taken.

The 60 patients who fulfilled the inclusion criteria were allocated to treatment according to a computer generated randomisation procedure with 15 consecutive balanced blocks of four patients (two active, two placebo). The figure shows details of the treatment periods and allocation. Table 1 shows the timing of

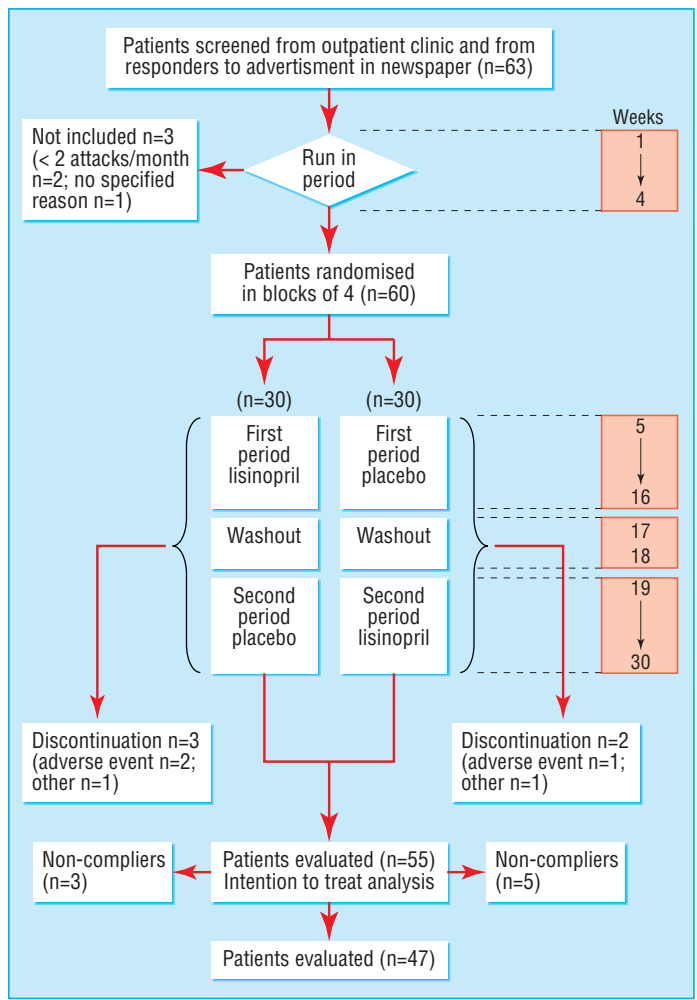

Trial profile in study of prophylactic treatment of migraine with lisinopril
Table 1 Timing of measures from baseline (week 1) to end of trial on effect of lisinopril on migraine

\begin{tabular}{|c|c|}
\hline Measures & Weeks \\
\hline $\begin{array}{l}\text { Participants visited; heart rate and blood pressure } \\
\text { measured }\end{array}$ & $1,5,7,17,21,31$ \\
\hline Participants telephoned & 12,26 \\
\hline Inclusion/exclusion criteria checked & 1,5 \\
\hline Somatic and neurological state checked & 1,31 \\
\hline Blood sample analysed & $1,7,21$ \\
\hline $\begin{array}{l}\text { Headache diaries and compliance checked; tablet delivery } \\
\text { and receipt; quality of life questionnaires completed }\end{array}$ & $1,5,17,31$ \\
\hline
\end{tabular}

measures throughout the study. The participants kept a daily diary in which they recorded the presence, duration in hours, and severity (mild, moderate, severe, excruciating) of headache; the presence and severity (mild, severe) of accompanying nausea, photophobia, and phonophobia; the use of symptomatic drugs; and sick leave. Participants stated whether the headache experienced was migraine or not. Quality of life was assessed with a standardised questionnaire (SF-36). ${ }^{4}$ After each treatment period participants were also asked about the acceptability of the treatment ("If you could receive this treatment on prescription, would you like to continue with the treatment that you have used in the past 12 weeks?”). Participants were defined as compliant with treatment if they had adhered to the drug regimen $(>80 \%$ of the tablets taken as determined by a tablet count at the end of the treatment period) and had given complete data in the diary. This was assessed by the study nurse and by the doctors.

Primary end point variables were number of hours with headache; number of days with headache, irrespective of duration and severity; and number of days with migraine, irrespective of duration and severity. Secondary end point variables were headache severity index, calculated by multiplying headache hours with the reported maximum severity that day (grade 1-4) and subsequently adding the results for all headache days during 12 weeks of either treatment period; doses of triptans and doses of analgesics; acceptability of treatment; days of sick leave; and health related quality of life variables (specifically, the SF-36 variables for quantification of bodily pain, general health, vitality, and social functioning).

\section{Statistical analysis}

Interaction effects were assessed by the Mann-Whitney $\mathrm{U}$ test. We used the Wilcoxon signed rank test to compare end point variables because one of the primary efficacy parameters (headache hours) was not normally distributed (Sapiro-Wilk $W$ test; $\mathrm{P}<0.0005$ ). For comparison of adverse events and acceptability we used a McNemar's matched pairs test. A two-sided $\mathrm{P}<0.05$ was considered significant. A paired study including 60 (or 55) subjects will have about 93\% (or $80 \%$ ) power to detect a group mean difference of 0.5 SD (with Student's $t$ test).

\section{Results}

\section{Participants}

To achieve the intended number of 60 randomised patients who met the inclusion and exclusion criteria we screened 63 patients in the placebo run-in period. 
Table 2 Adverse events in 60 participants with migraine treated with lisinopril or placebo*

\begin{tabular}{lcc} 
& Lisinopril & Placebo \\
\hline Coughing & 8 & 3 \\
\hline Fatigue & 3 & 3 \\
\hline Dizziness & 7 & 4 \\
\hline Tendency to faint & 3 & 0 \\
\hline Others & 3 & 3 \\
\hline Total & 24 & 13 \\
\hline
\end{tabular}

${ }^{\star} \mathrm{P}=0.07$ (McNemar's matched pairs test) for comparison of pooled effects: no adverse events $v$ at least one symptom $(2 \times 2$ table).

All participants completed this period and gave complete data in their diaries. Two were excluded from the study because they had fewer than two attacks, and one declined to participate without specifying the reason. Of the 60 patients who were randomised, three withdrew from the study because of adverse events (fatigue, dizziness and fatigue, exanthema, and monarthritis), one declined to continue, and one had an inadequate response on placebo (see figure). Eight patients did not comply with treatment (see box) but kept a diary for the whole study period. The 47 remaining participants (38 women, mean (SD) age 41 (9) years; nine men, 43 (5) years) provided complete data for final evaluation of efficacy. Table 2 shows the adverse events in the 60 randomised patients.

\section{Outcomes}

There were no significant interaction effects between periods as measured by the primary efficacy parameters. No parameter deviated significantly from a normal distribution except for hours with headache. Table 3 shows the effects of lisinopril. There was a significant difference in favour of lisinopril for hours with headache, number of days with headache, number of days with migraine, and headache severity index. In the intention to treat analysis in 55 patients, significant differences were retained for the primary efficacy end points (table 4).

In comparison with the baseline data from the four week run-in period, treatment with lisinopril resulted in reductions in hours with headache, days with headache, and days with migraine (table 5). A reduction of

\section{Details of non-compliance in eight participants} (treatment group shown in parentheses)

Shortening of second period to 11 weeks because of erroneous three week wash out period (active)

Diarrhoea, dizziness, nausea; reduction to one tablet daily after three weeks, no tablet intake in last week (active)

Low blood pressure, syncope, fatigue; no tablet intake in last two weeks (active)

Thought there was no effect and was uncomfortable; no tablet intake in last three weeks (active)

Erroneous intake of one tablet in whole period (placebo)

Thought there was no effect and was uncomfortable; no tablet intake in last week (placebo)

Erroneous intake of one tablet from second to sixth week (placebo)

Intake of one tablet in third week; no tablets in last seven weeks (active)
Table 3 Efficacy parameters in 47 participants with migraine during treatment periods of 12 weeks. Figures are means (SD)

\begin{tabular}{|c|c|c|c|}
\hline & Lisinopril & Placebo & $\begin{array}{l}\text { Mean \% reduction } \\
(95 \% \mathrm{Cl})\end{array}$ \\
\hline \multicolumn{4}{|c|}{ Primary efficacy parameter } \\
\hline Hours with headache & $129(125)$ & $162(142)$ & 20 (5 to 36$)$ \\
\hline Days with headache & $19.7(14)$ & $23.7(11)$ & 17 (5 to 30$)$ \\
\hline Days with migraine & $14.5(11)$ & $18.5(10)$ & 21 (9 to 34$)$ \\
\hline \multicolumn{4}{|c|}{ Secondary efficacy parameter } \\
\hline Headache severity index & $297(325)$ & $370(310)$ & 20 (3 to 37 ) \\
\hline Triptan doses & $15.7(15)$ & $20.2(17)$ & 22 (7 to 38$)$ \\
\hline Doses of analgesics & $14.5(23)$ & $16.2(20)$ & $11(-16$ to 37$)$ \\
\hline Days with sick leave & $2.30(4.32)$ & $2.09(2.50)$ & -10 (-64 to 37$)$ \\
\hline Bodily pain* & $63.7(29)$ & $53.8(23)$ & $-18(-35$ to -1$)$ \\
\hline General health* & $73.6(20)$ & $74.1(21)$ & $1(-6$ to 7$)$ \\
\hline Vitality* & $61.1(24)$ & $58.2(21)$ & $-5(-18$ to 8$)$ \\
\hline Social functioning* & $81.4(25)$ & $79.5(23)$ & $-2(-11$ to 6$)$ \\
\hline
\end{tabular}

${ }^{*}$ From SF-36.

Table 4 Intention to treat analysis of primary efficacy parameters in 55 participants during treatment periods of 12 weeks. Figures are means (SD)

\begin{tabular}{lccc} 
& Lisinopril & Placebo & $\begin{array}{c}\text { Mean \% reduction } \\
\text { (95\% CI) }\end{array}$ \\
\hline Hours with headache & $138(130)$ & $162(134)$ & $15(0$ to 30$)$ \\
\hline Days with headache & $20.7(14)$ & $24.7(11)$ & $16(5$ to 27$)$ \\
\hline Days with migraine & $14.6(10)$ & $18.7(9)$ & $22(11$ to 33$)$ \\
\hline
\end{tabular}

at least 50\% in symptoms was seen in $10(21 \%)$ participants for hours with headache, 13 (28\%) for days with headache, $14(30 \%)$ for days with migraine, and 15 $(32 \%)$ for headache severity index during lisinopril treatment compared with the placebo period. In comparison with the run-in period this degree of reduction was seen in $15(32 \%)$ for hours with headache, 16 (34\%) for days with headache, and $17(36 \%)$ for days with migraine.

Mean (SD) blood pressure was 128/83 (14/10) and mean (SD) heart rate was 71 (8) beats/min during the 12 week placebo period. During the lisinopril period mean blood pressure was 121/78 $(13 / 10)(\mathrm{P}<0.0001$ for systolic and diastolic pressure) and mean heart rate 69 (6) beats/min. Acceptability of treatment was 35/47 for lisinopril versus $14 / 47$ for placebo $(\mathrm{P}<0.0001)$. Except for bodily pain, which showed a reduction with lisinopril, health related quality of life scales showed no significant differences.

\section{Discussion}

Our results show that lisinopril has a clinically relevant prophylactic effect against migraine. Compared with baseline data (during run-in), there was a reduction of

Table 5 Primary efficacy parameters during run-in period (four weeks) compared with average during treatment with lisinopril or placebo in 47 participants

\begin{tabular}{|c|c|c|c|c|c|}
\hline & \multirow[b]{2}{*}{$\begin{array}{l}\text { Mean (SD) } \\
\text { in run in }\end{array}$} & \multicolumn{2}{|c|}{ Lisinopril } & \multicolumn{2}{|c|}{ Placebo } \\
\hline & & Mean (SD) & $\begin{array}{l}\text { Mean } \% \text { reduction } \\
\text { v run-in }(95 \% \mathrm{CI})\end{array}$ & Mean (SD) & $\begin{array}{c}\text { Mean } \% \\
\text { reduction } v \text { run- } \\
\text { in }(95 \% \mathrm{CI})\end{array}$ \\
\hline $\begin{array}{l}\text { Hours with } \\
\text { headache }\end{array}$ & $65(74)$ & $43(30)$ & 34 (10 to 57 ) & $54(32)$ & 17 (-1 to 35$)$ \\
\hline $\begin{array}{l}\text { Days with } \\
\text { headache }\end{array}$ & $9.4(4.0)$ & $6.6(4.0)$ & 30 (19 to 42$)$ & $7.9(3.0)$ & 16 (4 to 28 ) \\
\hline $\begin{array}{l}\text { Days with } \\
\text { migraine }\end{array}$ & $6.8(3.0)$ & $4.8(3.0)$ & 29 (15 to 42$)$ & $6.2(3.0)$ & 9 (-3 to 21$)$ \\
\hline
\end{tabular}


about $30 \%$ in primary efficacy parameters during lisinopril treatment. Compared with the placebo period, which is a more conservative approach, there was a reduction of about $20 \%$. A comparison of the effect of lisinopril with those reported for other prophylactic drugs for migraine is difficult because of differences in study designs and ways of reporting results. ${ }^{6}$ A meta-analysis of the effect of propranolol $160 \mathrm{mg}$ indicated an improvement of 33\% with regard to the headache index on active medication compared with placebo, but this analysis included both open and controlled studies. ${ }^{7}$ For this parameter we saw an improvement of $20 \%$, and we consider this to be a promising result in a study performed with an up to date and robust methodological design. To assess the relative efficacy, safety, and tolerability of different drugs reliably, however, only direct comparisons in a single study are valid.

\section{Study strengths}

The main strength of our study is that it was performed according to the guidelines for controlled trials of drugs in migraine. ${ }^{2}$ We chose the crossover design for this single centre study because fewer patients were needed than for a parallel group design. There are known disadvantages of the crossover design, ${ }^{8}$ but in this study we found no period effect and no carry over effect. The drop out rate was low $(8 \%)$, despite the relatively long duration of the study (31 weeks).

We did not use the "number of attacks" as an efficacy end point because participants would have had to record when the headache started and stopped. As the headache diary was already quite extensive we were concerned that this might cause a higher drop out rate. In addition, the frequent use of effective triptans modifies the attack pattern and makes it hard to assess the true attack rate as defined by the International Headache Society. Furthermore, we are not aware of any other studies that have detailed data on individual attacks such that the attack frequency could be reliably assessed according to the International Headache Society guidelines and comparisons made between studies. We therefore used the less ambiguous end points of number of days with migraine, number of days with headache, and number of hours with headache.

\section{Why it might work}

Lisinopril has various pharmacological effects that may be relevant in migraine. In addition to blocking the conversion of angiotensin I to angiotensin II, it also alters sympathetic activity, inhibits free radical activity, increases prostacyclin synthesis, ${ }^{9}$ and blocks the degradation of bradykinin, encephalin, and substance $\mathrm{P} .{ }^{10}$ Of great relevance may be the recent finding that migraine without aura seems to be more common in people with the angiotensin converting enzyme DD gene, and migraineurs with this gene also have higher angiotensin converting enzyme activity and a higher frequency of attacks than other migraine sufferers. ${ }^{11}$

\section{Safety and tolerability}

Lisinopril was well tolerated, as can be seen from the acceptability and the quality of life scores, and the adverse events observed in this study were those known to be associated with angiotensin converting

\section{What is already known on this topic}

Many drugs recommended for prophylaxis of migraine are not suitable for long term use

\section{What this study adds}

The angiotensin converting enzyme inhibitor lisinopril is an effective prophylactic treatment for frequent migraine attacks

Lisinopril significantly decreased hours with headache, days with headache, days with migraine, headache severity index, and doses of triptan

Lisinopril is well tolerated and adverse events are mild or moderate

enzyme inhibitors. ${ }^{12}$ Symptoms associated with hypotension (dizziness and tendency to faint) may be minimised by reducing the intake of lisinopril to its lowest effective dose. Cough, a side effect not related to dose, was severe enough to prohibit further use in only three patients.

In contrast with $\beta$ blockers, lisinopril can be used in patients with asthma, intermittent claudication, and conduction defects, and it is not associated with sexual dysfunction. ${ }^{13}$ Angiotensin converting enzyme inhibitors are known to cause fetal and neonatal morbidity and mortality in the second and third trimesters of pregnancy, ${ }^{14}$ but in contrast with valproic acid, lisinopril is considered relatively safe during organogenesis in the first trimester. This may allow for a cautious use in women of childbearing age as the drug can be discontinued when pregnancy is diagnosed.

Lisinopril is widely prescribed for various cardiovascular conditions and has a well established safety profile. Doctors are already familiar with prescribing angiotensin converting enzyme inhibitors. Thus, given the limitation of this being one relatively small study, albeit with a robust double blind and placebo controlled design, the positive outcomes and good tolerability support the use of lisinopril as a useful prophylactic treatment for migraine patients.

Contributors: HS had the original idea for the study and is the guarantor. HS and LJS were the principal investigators and designed the protocol, assessed the patients, and wrote the manuscript. GH assisted in the design of the study, recruitment of the patients, and data collection and analysis. TS helped to draw up the protocol and participated in the statistical analysis. TS and GH contributed to the revision of the manuscript. GB participated in planning the study, assessment of patients, and revision of the manuscript. He also advised on the study design and the statistical analysis. The database was managed by the investigators who performed the statistical analyses of the data.

Funding: AstraZeneca provided the computer generated randomisation scheme, study medication, and financial support.

Competing interests: HS and GB have been reimbursed by AstraZeneca, one of the manufacturers of lisinopril, for attending conferences. These conferences were unrelated to the present study.

1 Bender WI. ACE inhibitors for prophylaxis of migraine headaches. Headache 1995;35:470-1.

2 International Headache Society Committee on Clinical Trials in Migraine. Guidelines for controlled trials of drugs in migraine. Cephalalgia 1991;11/1:1-12.

3 Headache Classification Committee of the International Headache Society. Classification and diagnostic criteria for headache disorders, cranial neuralgias and facial pain. Cephalalgia 1988;8(suppl 7):1-96. 
4 Ware JE, Gandek B, IQOLA project group. The SF-36 health survey: development and use in mental health research and the IQOLA project. Int J Mental Health 1994;23:49-73.

5 Lachin JM. Introduction to sample size determination and power analysis in clinical trials. Control Clin Trials 1981;2:93-113.

6 Tfelt-Hansen P, Shanks RG. Beta-adrenoceptor blocking drugs. In Olesen J, Tfelt-Hansen P, Welch M, eds. The headaches. New York: Raven Press, 1993:363-72

7 Holroyd KA, Penzien DB, Cordingley GE. Propranolol in the management of recurrent migraine: a meta-analytic review. Headach 1991;31:33-40.

8 Altman DG. Practical statistics for medical research. London: Chapman and Hall, 1991:447-8.

9 Goa KL, Balfour JA, Zuanetti G. Lisinopril. A review of its pharmacology and clinical efficacy in the early management of acute myocardial infarction. Drugs 1996;52:564-88.

10 Skidgel RA, Erdos EG. The broad substrate specificity of human angiotensin converting enzyme. Clin Exp Hypertens A 1987;9:243-59.
11 Paterna S, Di Pasquale P, D'Angelo A, Seidita G, Tuttolomondo A, Cardinale A, et al. Angiotensin-converting enzyme gene deletion poly morphism determines an increase in frequency of migraine attacks in patients suffering from migraine without aura. Eur Neurol 2000;43;133-6.

12 Huckell VF, Belanger LG, Kazimirski M, Subramanian T, Cox AJ. Lisinopril in the treatment of hypertension: a Canadian postmarketing surveillance study. Clin Ther 1993;15:407-22.

13 Fogari R, Zoppi A, Corradi L, Mugellini A, Poletti L, Lusardi P. Sexual function in hypertensive males treated with lisinopril or atenolol: a crossover study. Am J Hypertens 1998;11:1244-7.

14 Tomlinson AJ, Campbell J, Walker JJ, Morgan C. Malignant primary hypertension in pregnancy treated with lisinopril. Ann Pharmacother 2000;34:180-2.

(Accepted 9 October 2000) 